\title{
Effect of diet, sex and age on fatty acid metabolism in broiler chickens: $\boldsymbol{n - 3}$ and $n-6$ PUFA
}

\author{
R. Poureslami ${ }^{1}$, K. Raes ${ }^{1} \dagger$, G. M. Turchini ${ }^{2}$, G. Huyghebaert ${ }^{3}$ and S. De Smet $^{1 *}$ \\ ${ }^{1}$ Laboratory for Animal Nutrition and Animal Product Quality, Department of Animal Production, Ghent University, \\ Proefhoevestraat 10, 9090 Melle, Belgium \\ ${ }^{2}$ School of Life and Environmental Sciences, Deakin University, Warrnambool, Vic, Australia \\ ${ }^{3}$ Animal Science Unit, Institute for Agricultural and Fisheries Research, Scheldeweg 68, 9090 Melle, Belgium \\ (Received 28 July 2009 - Revised 11 January 2010 - Accepted 20 January 2010 - First published online 1 March 2010)
}

The PUFA metabolism in broiler chicken was studied through the whole body fatty acid balance method. Four dietary lipid sources (palm fat, Palm; soyabean oil, Soya; linseed oil, Lin; fish oil, Fish) were added at $3 \%$ to a basal diet containing $5 \%$ palm fat. Diets were fed to female and male birds from day 1 to either day 21 or day 42 of age. Birds fed the Lin diet showed a significantly higher $18: 2 n-6$ accumulation compared with the other diets $(85.2$ v. $73.6 \%$ of net intake), whereas diet did not affect $18: 3 n-3$ accumulation (mean $63 \%$ of net intake). Bioconversion of $18: 2 n-6$ significantly decreased in the order Palm $>$ Lin $>$ Soya $>$ Fish $(4 \cdot 7,3.9,3.4$ and $1 \%$ of net intake, respectively). The $18: 3 n-3$ bioconversion on the Palm and Soya diets was similar and significantly higher than in broilers on the Lin diet $(9.1 \mathrm{v} .5 \cdot 8 \%$ of net intake). The $\beta$-oxidation of $18: 2 n-6$ was significantly lower on the Lin diet than on the other diets $(10.8 v .23 .3 \%$ of net intake), whereas $\beta$-oxidation of $18: 3 n-3$ was significantly higher on the Fish diet than on the other diets $(41.5$ v. $27 \cdot 3 \%$ of net intake). Feeding fish oil suppressed apparent elongase and desaturase activity, whereas a higher dietary supply of $18: 3 n-3$ and $18: 2 n-6$ enhanced apparent elongation and desaturation activity on the PUFA involved in the $n-3$ and $n-6$ pathway, respectively. Accumulation of $18: 2 n-6$ and $18: 3 n-3$ increased and $\beta$-oxidation decreased with age. Sex had a marginal effect on the PUFA metabolism.

\section{PUFA: Broiler chickens: Elongation: Desaturation: Oxidation}

In chickens, as with all animals, body fatty acids are derived from dietary uptake, de novo synthesis and/or bioconversion. Among the different fatty acid classes, $n-3$ long-chain PUFA ( $n$-3 LCPUFA) are of particular interest due to their beneficial role on human health ${ }^{(1)}$. In vertebrates, $\alpha$-linolenic acid $(18: 3 n-3)$ and linoleic acid $(18: 2 n-6)$ cannot be biosynthesised de novo, but are derived from the diet and subsequently can be bioconverted to longer and more unsaturated $n-3$ and $n$-6 LCPUFA, respectively. This in vivo bioconversion of $n-3$ LCPUFA includes endoplasmic $\Delta-6$ desaturation, chain elongation and $\Delta-5$ desaturation of the precursor $18: 3 n-3$ to EPA $(20: 5 n-3)$, which is subsequently converted to docosapentaenoic acid $(22: 5 n-3)$ by chain elongation. The final metabolite, DHA $(22: 6 n-3)$, is synthesised by chain elongation, $\Delta-6$ desaturation and peroxisomal $\beta$-oxidation of $22: 5 n-3^{(2)}$. The $n-6$ pathway involves the same enzymes and conversion steps as the $n-3$ pathway, with arachidonic acid (20:4n-6) being the major metabolite of dietary $18: 2 n-6$.

The absolute amount of $18: 3 n-3$ intake is of prime importance to the efficiency of conversion to the LCPUFA ${ }^{(3)}$. However, the production of $n-3$ LCPUFA, particularly $22: 6 n-3$, from $18: 3 n-3$ is limited in the human body ${ }^{(4,5)}$. Isotopic tracer studies showed that the conversion of $18: 3 n-3$ to $22: 6 n-3$ is about $1 \%$ in infants and substantially lower in adults with, however, important differences between men and women ${ }^{(4,5)}$. Therefore, it is often postulated that the $n-3$ LCPUFA are semi-essential and should be sufficiently provided by the diet. In order to optimise the fatty acid composition of foods derived from farmed animals, knowledge of their LCPUFA metabolism is required. To study PUFA metabolism, several in vivo and ex vivo methods have been applied in different species. Isolation of cells or tissue microsomes and incubation with labelled fatty acids is a common ex vivo method $^{(6)}$. Besides the need for sophisticated analyses and expensive reagents, other drawbacks of the ex vivo methods are that some controlling factors are missing and that this approach is restricted to one tissue ${ }^{(7)}$. Therefore, to gain an accurate insight into the whole body (WB) metabolism of PUFA, a proper in vivo method is desired. Different in vivo techniques appropriate to WB fatty acid metabolism have been detailed elsewhere ${ }^{(8)}$. Recently, a WB fatty acid balance method has been suggested as a reliable method to study the overall PUFA metabolism in fish ${ }^{(7)}$. The WB fatty acid balance method comprises a feeding experiment, quantification

Abbreviations: Fish, fish oil diet; LCPUFA, long-chain PUFA; Lin, linseed oil diet; Palm, palm fat diet; Soya, soyabean oil diet; WB, whole body.

* Corresponding author: Stefaan De Smet, fax +32 9264 9099, email stefaan.desmet@ugent.be

$\dagger$ Present address: Research Group EnBiChem, Department of Industrial Engineering and Technology, University College West-Flanders, Graaf Karel de Goedelaan 5, 8500 Kortrijk, Belgium 
and fatty acid methyl esters were analysed by GC (HP6890, Brussels, Belgium) on a CPSil88 column for fatty acid methyl esters $(100 \mathrm{~m} \times 0.25 \mathrm{~mm} \times 0.2 \mu \mathrm{m}$, Chrompack, Middelburg, The Netherlands) according to the method described by Raes et al. ${ }^{(11)}$. Fatty acids were identified by comparing their retention times with those of the corresponding standards (Sigma, Brussels, Belgium) Nonadecanoic acid was used as internal standard to obtain quantitative data of the fatty acid contents (mg/100g tissue). Before WB fatty acid balance calculations, a theoretical correction was made for fatty acid methyl esters based on the response correction factors discussed by Ackman ${ }^{(12)}$.

\section{Whole body fatty acid balance calculations}

The different fates (excretion, body accumulation, elongation, desaturation and mitochondrial $\beta$-oxidation) of dietary $18: 2 n-6$ and $18: 3 n-3$ were calculated according to the method described in detail by Turchini et al. ${ }^{(7,13)}$ and Turchini \& Francis $^{(14)}$. Summarising the methodology, the WB fatty acid balance was computed in distinct steps. Initially, the fatty acids in the diets, faeces and the initial and final carcasses (sum of fatty acid mass in seven body compartments) were quantified in $\mathrm{mg}$. Knowing the fatty acid mass ingested and excreted, apparent digestibility of individual fatty acids was computed, and the net fatty acid intake (fatty acid absorption) was calculated. The difference between fatty acid accumulation in the WB and the net intake was considered as the total appearance or disappearance of a specific fatty acid. Next, the balance of $n-3$ and $n-6$ PUFA was computed. The quantity of fatty acid was first converted from $\mathrm{mg}$ to $\mu \mathrm{mol}$ of appeared/disappeared fatty acid per chicken WB. Then a backward calculation was made along each of the fatty acid metabolic pathways. The number of $\mu \mathrm{mol}$ of a longer chain or more unsaturated fatty acid that appeared was subtracted from the number of $\mu$ mol of the previous fatty acid in the pathway. This allowed quantifying the bioconversion (i.e. elongation and/or desaturation) of an individual fatty acid expressed as $\mu \mathrm{mol}$ of fatty acid/g body weight per day. Fatty acid $\beta$-oxidation was subsequently computed after all computations along the pathway. In four cases (out of forty-eight), a small negative $\beta$-oxidation value was obtained (i.e. an appearance of $18: 2 n-6$ or $18: 3 n-3$ ). These values were turned from a negative value to zero before further calculation. It was verified that this did not affect the significance of the treatment effects. The equations and details for calculating the fate of individual fatty acids (\% ex novo production, $\%$ body accumulation, $\%$ bioconversion, $\% \beta$-oxidation) were previously described ${ }^{(14)}$.

\section{Data analyses}

The data were analysed using S-PLUS for Windows (version 6.1; Insightful, Seattle, WA, USA). A linear model was used to analyse the fixed effects of diet, age and sex and their interaction terms. In case of a significant diet effect, the mean values were compared with the Tukey's post hoc test $(P<0 \cdot 05)$.

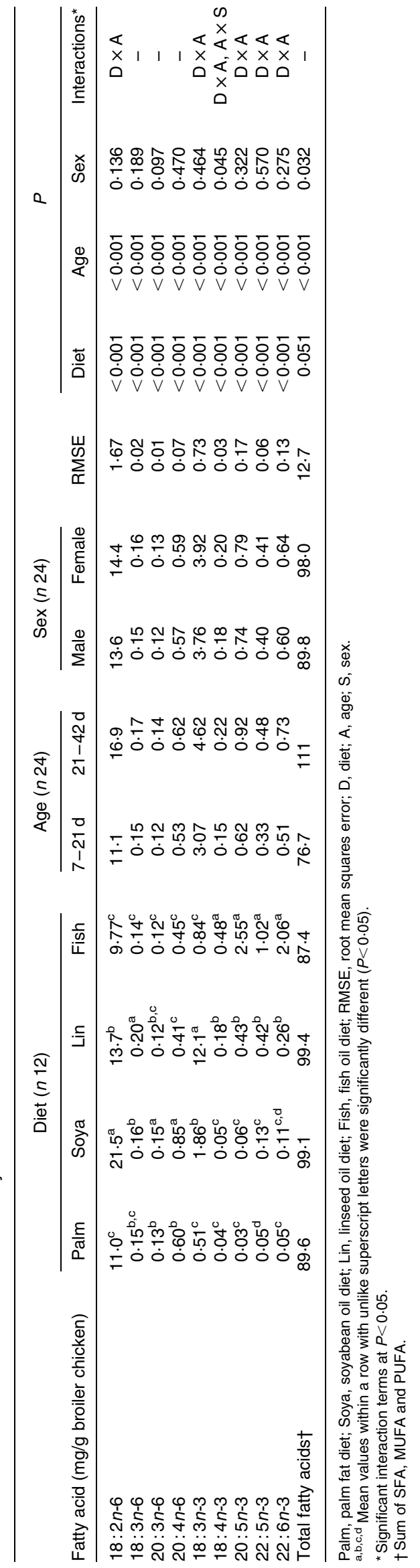


Table 4. $n-3$ and $n$-6 PUFA intake, apparent digestibility, appearance/disappearance, accumulation, bioconversion and $\beta$-oxidation in broiler chickens

\begin{tabular}{|c|c|c|c|c|c|c|c|c|c|c|c|c|c|}
\hline & \multicolumn{4}{|c|}{ Diet $(n 12)$} & \multicolumn{2}{|c|}{ Age $(n 24)$} & \multicolumn{2}{|c|}{$\operatorname{Sex}(n 24)$} & \multirow[b]{2}{*}{ RMSE } & \multicolumn{4}{|c|}{$P$} \\
\hline & Palm & Soya & Lin & Fish & $7-21 d$ & $21-42 d$ & Male & Female & & Diet & Age & Sex & Interactions ${ }^{*}$ \\
\hline \multicolumn{14}{|c|}{ Intake (mg/d) } \\
\hline $18: 2 n-6$ & $1552^{\mathrm{b}}$ & $2472^{\mathrm{a}}$ & $1555^{\mathrm{b}}$ & $1252^{\mathrm{c}}$ & 1026 & 2389 & 1834 & 1581 & 74.8 & $<0.001$ & $<0.001$ & $<0.001$ & $\mathrm{D} \times \mathrm{A}, \mathrm{D} \times \mathrm{S}, \mathrm{A} \times \mathrm{S}, \mathrm{D} \times \mathrm{A} \times \mathrm{S}$ \\
\hline $18: 3 n-3$ & $90 \cdot 8^{d}$ & $245^{\mathrm{b}}$ & $1508^{a}$ & $129^{c}$ & 292 & 695 & 527 & 459 & $33 \cdot 2$ & $<0.001$ & $<0.001$ & $<0.001$ & $D \times A, D \times S, A \times S, D \times A \times S$ \\
\hline $20: 5 n-3$ & - & - & - & 417 & 243 & 591 & 450 & 384 & $18 \cdot 0$ & - & $<0.001$ & $<0.001$ & - \\
\hline $22: 6 n-3$ & - & - & - & 307.5 & 179 & 436 & 331 & 283 & $13 \cdot 2$ & - & $<0.001$ & $<0.001$ & - \\
\hline \multicolumn{14}{|c|}{ Apparent digestibility (\%) } \\
\hline $18: 2 n-6$ & $69 \cdot 0^{\mathrm{b}}$ & $85 \cdot 0^{\mathrm{a}}$ & $73.4^{\mathrm{b}}$ & $73.0^{\mathrm{b}}$ & 73.0 & $77 \cdot 0$ & 75.4 & 74.6 & 4.46 & $<0.001$ & 0.003 & 0.551 & $D \times A, D \times S$ \\
\hline $18: 3 n-3$ & $62 \cdot 0^{\mathrm{c}}$ & $87 \cdot 0^{\mathrm{a}}$ & $91 \cdot 0^{\mathrm{a}}$ & $77 \cdot 0^{\mathrm{b}}$ & 77.5 & $81 \cdot 0$ & $80 \cdot 0$ & $78 \cdot 0$ & 4.56 & $<0.001$ & 0.016 & 0.361 & $\mathrm{D} \times \mathrm{S}$ \\
\hline $20: 5 n-3$ & - & - & - & 93.5 & $90 \cdot 8$ & $96 \cdot 2$ & $92 \cdot 0$ & $95 \cdot 0$ & 3.95 & - & 0.047 & 0.225 & - \\
\hline $22: 6 n-3$ & - & - & - & $92 \cdot 8$ & $89 \cdot 8$ & 95.8 & 91.3 & 94.3 & 3.92 & - & 0.029 & 0.222 & - \\
\hline \multicolumn{14}{|c|}{ Total appearance/disappearance ( $\mu \mathrm{mol} / \mathrm{g}$ per d) } \\
\hline $18: 2 n-6$ & $-1.17^{\mathrm{b}}$ & $-2.09^{\mathrm{a}}$ & $-0.59^{b}$ & $-1.01^{b}$ & -1.92 & -0.51 & -1.29 & $-1 \cdot 14$ & 0.58 & $<0.001$ & $<0.001$ & 0.395 & $\mathrm{D} \times \mathrm{A}$ \\
\hline $18: 3 n-6$ & $0.028^{b}$ & $0.031^{b}$ & $0.047^{a}$ & $0.002^{c}$ & 0.029 & 0.024 & 0.028 & 0.025 & 0.008 & $<0.001$ & 0.039 & 0.181 & $\mathrm{D} \times \mathrm{A}, \mathrm{A} \times \mathrm{S}$ \\
\hline $20: 3 n-6$ & $0.02^{b}$ & $0.03^{\mathrm{a}}$ & $0.02^{b}$ & $0.01^{c}$ & 0.03 & 0.02 & 0.03 & 0.03 & 0.003 & $<0.001$ & $<0.001$ & 0.929 & $D \times A$ \\
\hline $20: 4 n-6$ & $0.13^{\mathrm{b}}$ & $0.20^{\mathrm{a}}$ & $0.09^{c}$ & $0.02^{\mathrm{d}}$ & 0.13 & 0.09 & 0.11 & 0.11 & 0.02 & $<0.001$ & $<0.001$ & 0.982 & $D \times A$ \\
\hline $18: 3 n-3$ & $-0.08^{b}$ & $-0.30^{\mathrm{b}}$ & $-1.81^{a}$ & $-0.17^{b}$ & -0.82 & -0.36 & -0.58 & -0.60 & 0.25 & $<0.001$ & $<0.001$ & 0.793 & $D \times A$ \\
\hline $18: 4 n-3$ & $-0.003^{c}$ & $0.007^{\mathrm{c}}$ & $0.043^{b}$ & $-0.156^{\mathrm{a}}$ & -0.037 & -0.017 & -0.030 & -0.024 & 0.012 & $<0.001$ & $<0.001$ & 0.094 & $D \times A$ \\
\hline $20: 5 n-3$ & $0.01^{\mathrm{c}}$ & $0.01^{c}$ & $0 \cdot 10^{\mathrm{a}}$ & $-0.75^{\mathrm{b}}$ & -0.20 & -0.11 & -0.16 & -0.15 & 0.05 & $<0.001$ & $<0.001$ & 0.488 & $D \times A$ \\
\hline $22: 5 n-3$ & $0.01^{\mathrm{C}}$ & $0.03^{b}$ & $0.09^{\mathrm{a}}$ & $0.09^{\mathrm{a}}$ & 0.06 & 0.05 & 0.05 & 0.05 & 0.01 & $<0.001$ & 0.519 & 0.857 & $D \times A$ \\
\hline $22: 6 n-3$ & $0.01^{\mathrm{c}}$ & $0.02^{\mathrm{c}, \mathrm{b}}$ & $0.05^{\mathrm{b}}$ & $-0.46^{\mathrm{a}}$ & -0.12 & -0.07 & -0.09 & -0.09 & 0.03 & $<0.001$ & $<0.001$ & 0.366 & $D \times A$ \\
\hline \multicolumn{14}{|c|}{ Accumulation (\% of net intake) } \\
\hline $18: 2 n-6$ & $73.1^{\mathrm{b}}$ & $74.7^{\mathrm{b}}$ & $85 \cdot 2^{\mathrm{a}}$ & $73.0^{\mathrm{b}}$ & $67 \cdot 2$ & $85 \cdot 8$ & $74 \cdot 8$ & $78 \cdot 2$ & $8 \cdot 80$ & 0.004 & $<0.001$ & 0.195 & - \\
\hline $18: 3 n-3$ & 63.9 & $64 \cdot 3$ & $65 \cdot 3$ & 58.4 & $53 \cdot 8$ & $72 \cdot 2$ & $60 \cdot 8$ & $65 \cdot 2$ & $9 \cdot 87$ & 0.329 & $<0.001$ & 0.134 & - \\
\hline \multicolumn{14}{|c|}{ Bioconversion (\% of net intake) } \\
\hline $18: 2 n-6$ & $4 \cdot 69^{\mathrm{a}}$ & $3.38^{\mathrm{C}}$ & $3.88^{\mathrm{b}}$ & $0.96^{\mathrm{d}}$ & $3 \cdot 17$ & $3 \cdot 28$ & $3 \cdot 24$ & $3 \cdot 21$ & 0.44 & $<0.001$ & 0.399 & 0.852 & - \\
\hline $18: 3 n-3$ & $9 \cdot 12^{\mathrm{a}}$ & $9 \cdot 12^{\mathrm{a}}$ & $5 \cdot 80^{\mathrm{b}}$ & $0.00^{C}$ & $5 \cdot 74$ & $6 \cdot 33$ & 5.42 & 6.65 & 1.70 & $<0.001$ & 0.238 & 0.018 & - \\
\hline \multicolumn{14}{|c|}{$\beta$-Oxidation (\% of net intake) } \\
\hline $18: 2 n-6$ & $22 \cdot 1^{\mathrm{a}}$ & $21 \cdot 8^{\mathrm{a}}$ & $10 \cdot 8^{\mathrm{b}}$ & $25 \cdot 9^{\mathrm{a}}$ & 29.5 & $10 \cdot 8$ & $21 \cdot 8$ & 18.5 & 8.85 & 0.001 & $<0.001$ & 0.201 & - \\
\hline $18: 3 n-3$ & $26 \cdot 7^{\mathrm{a}}$ & $26 \cdot 5^{a}$ & $28 \cdot 8^{\mathrm{a}}$ & $41.5^{\mathrm{b}}$ & $40 \cdot 3$ & $21 \cdot 4$ & 33.7 & $28 \cdot 1$ & $9 \cdot 37$ & 0.001 & $<0.001$ & 0.046 & - \\
\hline $20: 5 n-3$ & - & - & - & 0.66 & 0.87 & 0.45 & 0.68 & 0.65 & 0.12 & - & $<0.001$ & 0.671 & - \\
\hline $22: 6 n-3$ & - & - & - & 0.46 & 0.59 & 0.34 & 0.48 & 0.45 & 0.06 & - & $<0.001$ & 0.550 & - \\
\hline
\end{tabular}

Palm, palm fat diet; Soya, soyabean oil diet; Lin, linseed oil diet; Fish, fish oil diet; RMSE, root mean squares error; D, diet; A, age; S, sex

a,b,c,d Mean values within a row for diets with unlike superscript letters were significantly different $(P<0.05)$.

${ }^{*}$ Significant interaction terms at $P<0.05$. 


\section{Results}

In Table 3, the $n-3$ and n-6 PUFA content of WB broiler chicken is given. Feeding the Soya diet resulted in higher contents of $18: 2 n-6,20: 3 n-6$ and $20: 4 n-6$ compared with the three other diets $(P<0 \cdot 001)$, whereas the Lin diet induced the highest $18: 3 n-6$ and $18: 3 n-3$ contents compared with the other diets $(P<0 \cdot 001)$. The $18: 3 n-3$ content in Lin fed birds was 11 -fold greater than the average of the other dietary treatments $(P<0 \cdot 001)$. Birds fed the Fish diet recorded a substantially higher $n-3$ LCPUFA content (20:5n-3, 22:5n-3 and $22: 6 n-3)$ compared with the other diets $(P<0 \cdot 001)$. The $20: 5 n-3,22: 5 n-3$ and $22: 6 n-3$ content in Fish fed birds was $14 \cdot 3-, 8 \cdot 4$ - and 5-fold greater compared with the average of the non-Fish fed birds. There was a trend for an effect of diet on the total body fatty acid content $(P=0.051)$, with lower values for the Palm and Fish diets compared with the Soya and Lin diets. As expected, age and sex had a significant effect on the total body fatty acid content.

PUFA intake, apparent digestibility, total appearance/disappearance and fate of $18: 2 n-6$ and $18: 3 n-3$ are presented in Table 4. Apparent digestibility for $18: 2 n-6$ was significantly higher for Soya fed birds compared with the other diets $(P<0.001)$. Apparent digestibility of $18: 3 n-3$ was higher on the Soya and Lin diets than on the Palm and Fish diets $(P<0.001)$.

Disappearance of PUFA may imply elongation and desaturation to longer chain metabolites or utilisation of their carbon skeleton through $\beta$-oxidation for energy production. Disappearance of $18: 2 n-6$ and appearance of $20: 4 n-6$ were higher in Soya fed birds in contrast with the other dietary treatments $(P<0 \cdot 001)$. Disappearance of $18: 3 n-3$ and appearance of $18: 4 n-3$ and $20: 5 n-3$ were higher in Lin fed birds compared with the other diets $(P<0.001)$. Appearance of 22:5n-3 was similar for the Lin and Fish fed birds and higher than for the Palm and Soya diets $(P<0 \cdot 001)$. Appearance of $22: 6 n-3$ was higher on the Lin diet than on the Soya and Palm diets $(P<0.05$ only for difference Lin $v$. Palm diet). Birds of the Fish group recorded a net disappearance of $18: 4 n-3,20: 5 n-3$ and $22: 6 n-3(P<0 \cdot 001)$.

Birds fed the Lin diet had a higher $18: 2 n-6$ accumulation (\% of net intake) compared with the other diets $(P<0.01)$. Bioconversion (\% of net intake) of $18: 2 n-6$ to longer chain/ more unsaturated fatty acids decreased in the order Palm $>$ Lin $>$ Soya $>$ Fish $(P<0.001)$. The $18: 2 n-6 \beta$-oxidation $(\%$ net intake) on the Lin diet was 2 -fold lower than on the other diets $(P<0 \cdot 001)$. Accumulation of $18: 3 n-3$ was not significantly different among the diets. Birds fed the Fish diet did not demonstrate bioconversion of $18: 3 n-3$ to $18: 4 n-3$. However, $18: 3 n-3$ bioconversion on the Palm and Soya diets was similar and 1.5-fold higher than on the Lin diet $(P<0.001)$. The 18:3n-3 $\beta$-oxidation on the Fish diet was 1.4-fold higher than on the other dietary treatments $(P<0.001)$.

The effect of age was significant for PUFA intake, apparent digestibility, appearance/disappearance (except of $22: 5 n-3$ ) and the proportions of the net intake of $18: 2 n-6$ and $18: 3 n-3$ accumulated and $\beta$-oxidised. Birds slaughtered at day 42 of age, when compared with the $7-14 \mathrm{~d}$ age period, had higher values for PUFA intake, PUFA apparent digestibility and $18: 2 n-6$ and $18: 3 n-3$ accumulation, but lower values for PUFA appearance/disappearance and $\beta$-oxidation. The effect

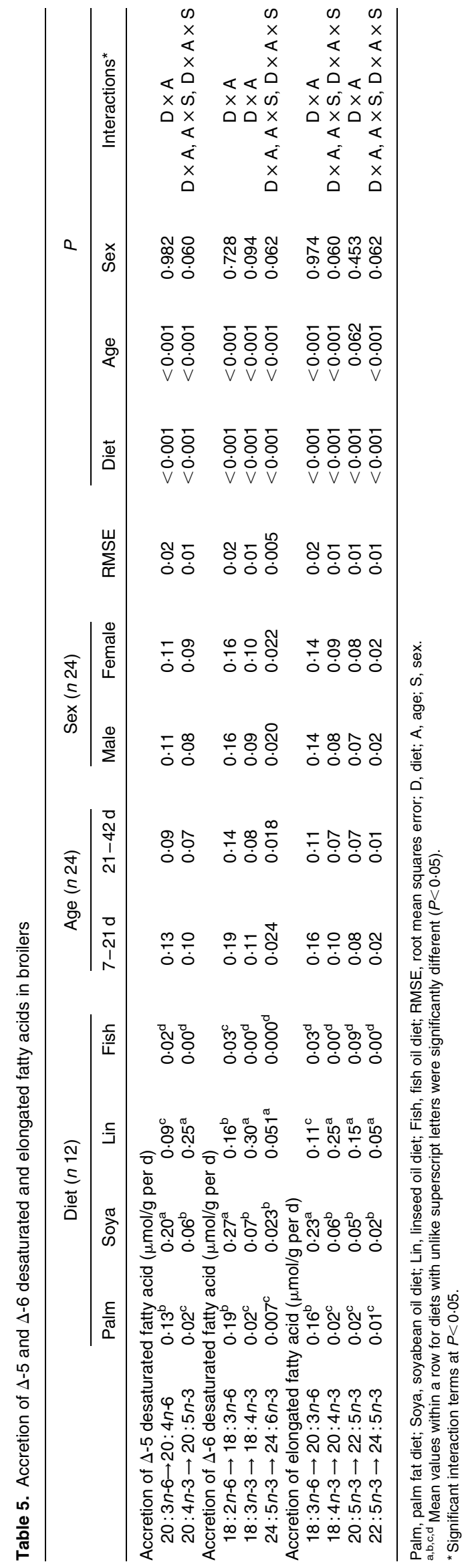


of sex on the above-mentioned variables was significant for $18: 2 n-6$ and $18: 3 n-3$ intake, $18: 3 n-3$ bioconversion and $18: 3 n-3 \quad \beta$-oxidation. Males had a greater $18: 2 n-6$ and $18: 3 n-3$ intake and $18: 3 n-3 \beta$-oxidation $(P<0 \cdot 05)$, whereas females showed a higher bioconversion of $18: 3 n-3$ to $18: 4 n-3(P<0 \cdot 05)$.

Accretion of $\Delta-5$ and $\Delta-6$ desaturated fatty acids and accretion of elongated fatty acids are presented in Table 5. Conversion of $20: 3 n-6$ to $20: 4 n-6$ as an indication of apparent $\Delta-5$ desaturation activity and conversion of $18: 2 n-6$ to $18: 3 n-6$ as an indication of apparent $\Delta-6$ desaturation activity in the $n-6$ pathway were higher in Soya fed birds compared with the other dietary treatments $(P<0.001)$. The $\Delta-5$ desaturation of $20: 4 n-3$ to $20: 5 n-3$ was higher in Lin fed birds compared with the other diets $(P<0 \cdot 001)$. Similarly, $\Delta-6$ desaturation of $18: 3 n-3$ to $18: 4 n-3$ and $22: 5 n-3$ to $22: 6 n-3$ was greater on the Lin diet compared with the other diets $(P<0.001)$. Elongation of $18: 3 n-6$ towards $20: 3 n-6$ was higher in Soya fed birds than in the other diets $(P<0 \cdot 001)$, and elongation of $18: 4 n-3,20: 5 n-3$ and $22: 5 n-3$ was higher in birds fed the Lin diet than in the other diets $(P<0 \cdot 001)$. Apparent desaturation activity was higher at $7-21 \mathrm{~d}$ of age than at $21-42 \mathrm{~d}$ of age $(P<0 \cdot 001)$. Apparent elongation activity on $18: 3 n-6$, $18: 4 n-3$ and $22: 5 n-3$ was higher in birds slaughtered on day 21 compared with day $42(P<0 \cdot 001)$, whereas elongation of $20: 5 n-3$ to $22: 5 n-3$ was not affected by age $(P>0 \cdot 05)$. Sex had no influence on the above-mentioned variables $(P>0 \cdot 05)$.

The apparent $\Delta-6$ desaturase activity acting on $18: 3 n-3$ and $18: 2 n-6$ was plotted against their respective net intake both expressed as $\mu \mathrm{mol} / \mathrm{g}$ per d (Figs 1 and 2). The apparent $\Delta-6$ desaturase activity on $18: 3 n-3$ showed a strong positive linear relationship with the $18: 3 n-3$ net intake $\left(R^{2} 0 \cdot 98\right)$, indicating that the higher the dietary $18: 3 n-3$ supply, the higher the resultant product $(18: 4 n-3)$. The response of apparent $\Delta$-6 desaturase activity to $18: 2 n-6$ net intake was positive but curvilinear $\left(R^{2} 0 \cdot 66\right)$, showing that by enhancing substrate $(18: 2 n-6)$ availability, the efficiency of $\Delta-6$ desaturase (18:3n-6 production) increased only up to a certain level. The $\Delta-5$ desaturase activity on $20: 4 n-3$ manifested a strong positive relationship $\left(R^{2} 0.99\right)$ with the summed $18: 4 n-3$ and $18: 3 n-3$ dietary supply (Fig. 3).

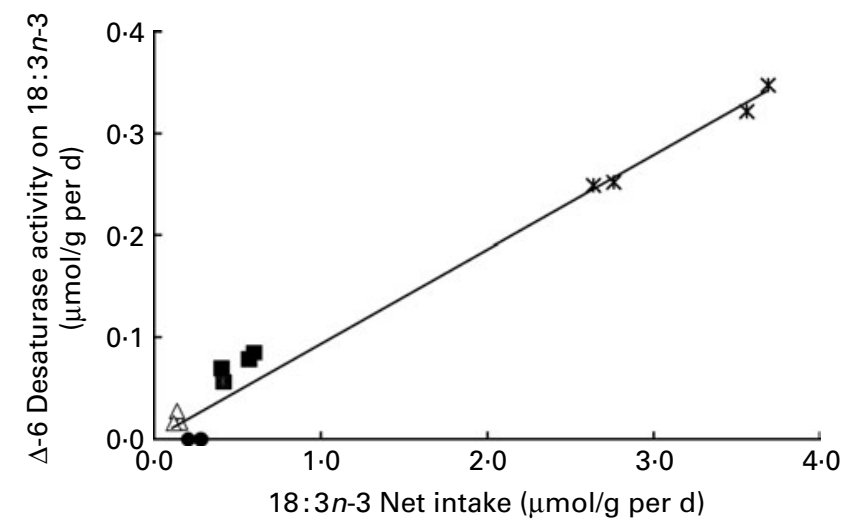

Fig. 1. Apparent $\Delta-6$ desaturase activity on $18: 3 n-3$ in relation to the $18: 3 n-3$ net intake $(\mu \mathrm{mol} / \mathrm{g}$ per $\mathrm{d})$ across four diets, age and sex. Linear regression equation: $Y=0.0030+0.0919 X ; R^{2} 0.98$; root mean squares error $=0.019$ ( $n$ 16). $\bullet$, Fish oil diet; $*$, linseed oil diet; $\mathbf{\square}$, soyabean oil diet; $\Delta$, palm fat diet.

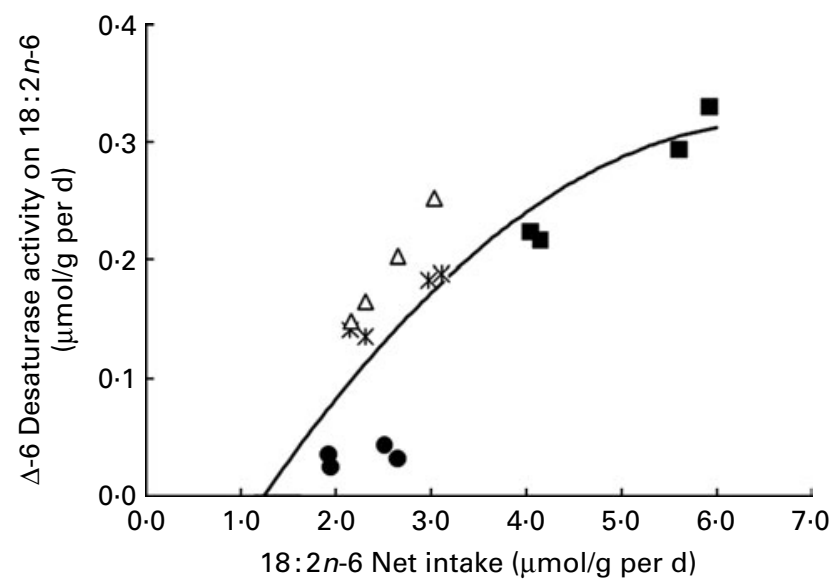

Fig. 2. Apparent $\Delta-6$ desaturase activity on $18: 2 n-6$ in relation to the $18: 2 n-6$ net intake ( $\mu \mathrm{mol} / \mathrm{g}$ per $\mathrm{d}$ ) across four diets, age and sex. Broken-line quadratic regression equation: $Y=0.3167$ if $X>6.669$ and $Y=0.3167-0.0107 \times$ $(6.669-X)^{2}$ if $X \leq 6.669 ; R^{2} 0.66$; root mean squares error $=0.058(n 16)$.

๑, Fish oil diet; $*$, linseed oil diet; $\mathbf{\square}$, soyabean oil diet; $\Delta$, palm fat diet.

Significant interaction terms are mentioned in the tables, but these effects are not further discussed here since they were negligible compared with the main effects and did not compromise the main conclusions. Most of the significant interaction effects were diet $\times$ age effects. $P$-values for the interaction effects were considerably smaller compared with the main effects. In addition, the significant interaction effects were the result of scale differences in most cases, i.e. the difference between the two age groups differed among diets in magnitude but not in sign (and vice versa) in case of a significant diet $X$ age effect. In few cases, the effect of age was opposite or absent in one diet versus the other diets, mostly when very low values occurred for a diet. The only marked diet $\times$ age interaction effect was for the digestibility of $18: 2 n-6$ and $18: 3 n-3$, which was approximately $10 \%$ lower in the young compared with the older birds on the Palm diet, whereas the age effect was negligible on the other diets.

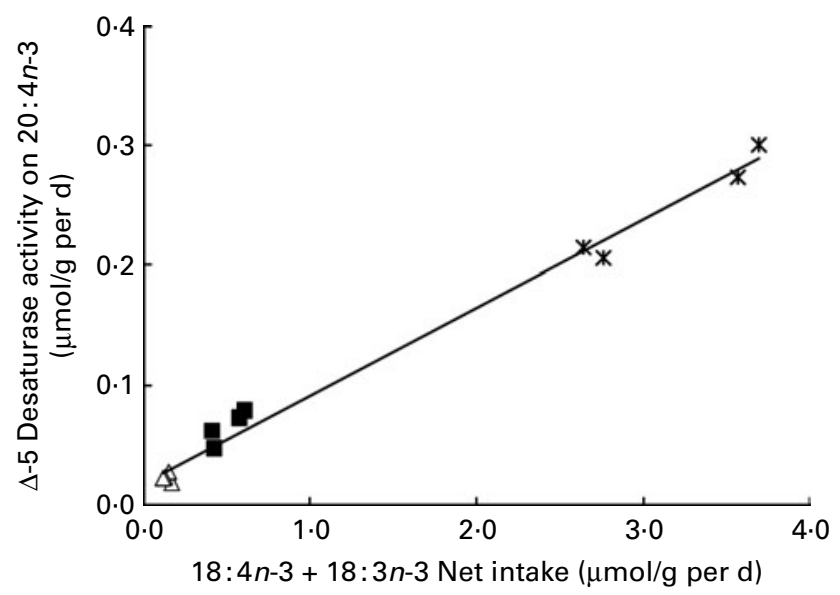

Fig. 3. Apparent $\Delta-5$ desaturase activity on $20: 4 n-3$ in relation to the $18: 4 n-3+18: 3 n-3$ net intake ( $\mu \mathrm{mol} / \mathrm{g}$ per d) across three diets, age and sex. Linear regression equation: $Y=0.0197+0.0728 X ; R^{2} 0.99$; root mean squares error $=0.011(n 12) . *$, linseed oil diet; $\boldsymbol{\square}$, soyabean oil diet; $\Delta$, palm fat diet. 


\section{Discussion}

The effect of diet and age on the fatty acid apparent digestibility in the present study is in agreement with previous studies in broiler chickens ${ }^{(15-17)}$. Many of the physiological functions necessary for lipid digestion are immature at hatch and develop over the next weeks. Bile salt secretion appears to be the first limiting factor for lipid digestion through the first few weeks post hatch ${ }^{(15)}$. In the present experiment, the apparent digestibility values of $18: 2 n-6,18: 3 n-3$ and $20: 5 n-3$ are in the same range as recently reported specifically for broiler chickens ${ }^{(17)}$. An earlier study described higher animal fat digestibility in female compared with male chickens $^{(15)}$. A slightly higher PUFA digestibility in female birds fed the Fish diet was observed in the present study; however, this difference was not statistically different. It should also be kept in mind that the digestibilities in the present experiment may be overestimated due to PUFA microbial biohydrogenation in the hindgut ${ }^{(17)}$.

The ingested PUFA face one of the three fates in the body, i.e. accumulation, conversion to longer chain/more unsaturated metabolites or $\beta$-oxidation. The WB fatty acid balance method allows quantifying the fate of the WB $18: 2 n-6$ and $18: 3 n-3$ relative to the net intake. Irrespective of the dietary treatment, broiler chickens seem to have a preference for accumulation of $18: 2 n-6$ above $18: 3 n-3$. Reviewing the literature, quantitative data on fatty acid metabolism in broiler chicken are scarce. Accumulation of $18: 2 n-6$ and $18: 3 n-3$ in WB level of growing pig fed a maize-soyabean meal basal diet (without added fat) was approximately 67 and $48 \%$ of digestible fatty acid, respectively ${ }^{(18)}$. In a WB fatty acid study with Murray cod fish fed with Lin, 40 and $53 \%$ accumulation of net intake for $18: 2 n-6$ and $18: 3 n-3$ were respectively reported ${ }^{(19)}$. These values are lower than for the $18: 2 n-6$ and $18: 3 n-3 \%$ net intake accumulation in Lin fed broilers in the present study. In a recent study with rainbow trout fed with fish oil, 91 and $60 \%$ net intake of $18: 2 n-6$ and $18: 3 n-3$ were respectively deposited in the $\mathrm{WB}^{(14)}$. However, in Lin fed trout, the corresponding values were 70 and $58 \%$ of net intake for $18: 2 n-6$ and $18: 3 n-3$, respectively. Therefore, it is possible to speculate that comparing chicken with rainbow trout both fed with fish oil, $18: 2 n-6$ and $18: 3 n-3$ accumulation is greater in fish than in chicken. Consequently, $18: 2 n-6$ and $18: 3 n-3$ accumulation appears greater in chicken compared with fish when fed Lin.

Across the four dietary treatments, the conversion rate of $18: 3 n-3$ to $18: 4 n-3$ was $1 \cdot 8$-fold higher than the conversion rate of $18: 2 n-6$ to $18: 3 n-6$ indicating a higher affinity of $\Delta-6$ desaturase for $18: 3 n-3$ than for $18: 2 n-6$. A relatively lower $18: 3 n-3$ bioconversion and increased $\beta$-oxidation rate in the Lin diet in contrast with Palm and Soya fed birds are in agreement with a previous report by Vermunt et al. ${ }^{(20)}$, stating that diets rich in $18: 3 n-3$ increase $18: 3 n-3$ $\beta$-oxidation, coincident with decreasing its conversion rate to longer and more unsaturated $n$-3 LCPUFA. In a WB fatty acid study with Murray cod fish fed Lin diet, 4 and $8 \%$ of $18: 2 n-6$ and $18: 3 n-3$ net intake were respectively converted to longer chain metabolites ${ }^{(19)}$. Hence, it seems there is a larger 18:3n-3 conversion in Lin fed Murray cod fish than in Lin fed chicken in our experiment. Similar to our findings, rainbow trout fed with fish oil did not demonstrate conversion of $18: 3 n-3$ to $18: 4 n-3$ in $\mathrm{WB}^{(14)}$. Feeding vegetable oil rather than fish oil increases desaturase and elongase activity as well as upregulates liver fatty acid elongase and FAD5 gene expression $^{(14,21)}$. This explains the higher conversion of $18: 3 n-3$ to $18: 4 n-3$ in the Palm, Soya and Lin diets (5- to 9-fold) compared with the Fish diet in the present study. The 18:2n-6 bioconversion in rainbow trout fed with fish oil $^{(14)}$ was $1 \cdot 8$-fold higher than in chicken fed fish oil in the present study. Comparing rainbow trout fish fed with Lin with the Lin treatment in our experiment indicated that the $18: 2 n-6$ conversion was $1 \cdot 2$-fold higher in chicken albeit the $18: 3 n-3$ conversion was 2 -fold higher in fish.

The higher $18: 3 n-3$ bioconversion in female birds of the present study is in agreement with previous findings in other species, e.g. human and rat ${ }^{(22,23)}$. Both the role of steroid hormones and other sex differences in metabolism may be involved. The $n-3$ LCPUFA content in plasma and tissues of rats appear to be positively related to circulating concentrations of oestradiol and progesterone and negatively associated with circulating concentrations of testosterone ${ }^{(24)}$, and testosterone administration to rats strongly depressed $\Delta-5$ and $\Delta-6$ desaturase activity ${ }^{(22)}$. In addition, fat $\beta$-oxidation was found to be greater in men than in women in several studies $^{(23,25)}$, corresponding to our finding that $\beta$-oxidation of $18: 2 n-6$ and $18: 3 n-3$ was higher in males than in females. Therefore, the availability of $18: 3 n-3$ for conversion to metabolites may be higher in females than in males. However, caution is needed when comparing the present results with those from studies involving mature animals or human subjects, since the male chickens in the present study were still immature. It is thus not clear whether the observed differences are mainly due to an effect of sex hormones or due to differences in energy metabolism.

The WB fatty acid balance method applied in the present study provided an estimation of apparent in vivo $\Delta-5$ and $\Delta-6$ desaturase activity. Regression analysis demonstrated a linear response in $\Delta-6$ desaturase activity in the $n-3$ pathway to an increasing supply of the substrate $18: 3 n-3$. The same trend was observed for $\Delta-5$ desaturase activity. On the other hand, the response in $\Delta-6$ desaturase activity in the $n-6$ pathway to an increasing supply of $18: 2 n-6$ was curvilinear. However, it should be noted that the range of net intake of $18: 2 n-6$ was larger than for $18: 3 n-3$, and that the curvilinear response could be partly due to the considerably lower $\Delta-6$ desaturase activity on the Fish diet compared with a similar $18: 2 n-6$ net intake values on the other diets.

Crespo \& Esteve-Garcia ${ }^{(16)}$ reported that the total $\beta$-oxidation of $n$ - 6 PUFA was 29 and $11 \%$ of net intake, and 29 and $14 \%$ for $n-3$ PUFA, in linseed and sunflower oil fed broiler chickens, respectively. However, the individual fates of $18: 2 n-6$ and $18: 3 n-3$ were not reported. In growing pigs fed a maize-soyabean meal diet, $30 \%$ of the digested $\Sigma n-6$ fatty acid and $52 \%$ of the digested $18: 3 n-3$ were $\beta$-oxidised at WB level ${ }^{(18)}$. The $18: 3 n-3 \beta$-oxidation reported in the present study was $31 \%$ (across diets, age and sex), and therefore it seems to be lower in broiler chickens in contrast with swine. In rat, Leyton et al. ${ }^{(26)}$ measured exhalation of ${ }^{14} \mathrm{CO}_{2}$ and demonstrated that the $\beta$-oxidation rate of $18: 2 n-6$ and $18: 3 n-3$ was 48 and $64 \%$, respectively. In human, over $30 \%$ of labelled $18: 3 n-3$ administered were used as energy source in man; however, lower values (22\%) were reported 
in women ${ }^{(23,27)}$. Consistently, numerically lower $\beta$-oxidation values for $18: 2 n-6$ and $18: 3 n-3$ were also recorded in female chickens in the present study.

In fish, the $\beta$-oxidation of $18: 2 n-6$ and $18: 3 n-3$ was 29 and $8 \%$ of net intake, respectively, at WB level of Murray cod fish fed with $\operatorname{Lin}^{(19)}$; while in rainbow trout fed with fish oil, $18: 2 n-6$ and $18: 3 n-3 \quad \beta$-oxidation was reported as 7 and $40 \%$ of net intake ${ }^{(14)}$. Comparing our findings with the latter study, which was implemented with similar methodologies, reveals that $18: 2 n-6 \beta$-oxidation in fish oil fed chicken is about 3 - to 4-fold higher than in rainbow trout, while $18: 3 n-3 \beta$-oxidation is approximately similar in the two species (40v. $41 \%$ of net intake). In Lin fed rainbow trout, the $\beta$-oxidation of $18: 2 n-6$ and $18: 3 n-3$ was 26 and $29 \%$ of the net intake ${ }^{(14)}$. This suggests that $18: 2 n-6$ $\beta$-oxidation in linseed fed chicken is 3 -fold lower than in linseed fed rainbow trout. On the other hand, $18: 3 n-3$ $\beta$-oxidation in linseed fed chicken from the present study corresponds with linseed fed rainbow trout fish $(29 \%$ net intake). It is at this point important to highlight some fundamental differences between fish and birds basic nutrition: fish are cold-blooded animals and have minor metabolic energy requirements (i.e. no energetic costs for maintaining the position and minor energetic costs for the detoxification of $\mathrm{N}$ containing compounds). Birds derive the majority of their energy requirements from dietary carbohydrates; while in fish, carbohydrates are very poorly utilised. Accordingly, the lipid content of diets for the two groups of animals is remarkably different (approximately $8 \%$ in broiler chickens and approximately $25 \%$ in trout). Despite these important differences, our comparisons depicted earlier are relative to the fate of specific fatty acids as percentage of net intake, and therefore these differences have to be considered levelled. Thus, these comparisons are sound and useful in providing a better understanding of PUFA metabolism in different farmed animals.

The stage of the growth and development influences fatty acid $\beta$-oxidation ${ }^{(28)}$. In our experiment, $\beta$-oxidation of $18: 2 n-6, \quad 18: 3 n-3,18: 4 n-3,20: 5 n-3$ and $22: 6 n-3$ was higher in $21 \mathrm{~d}$-old birds compared with $42 \mathrm{~d}$-old birds. This corresponds with the fact that young fast growing chickens have a higher metabolism rate compared with the same birds approaching slaughter age.

The WB fatty acid balance method provides information about the overall fatty acid metabolism in the body. The method is comparatively inexpensive, easy and feasible in animal research laboratories ${ }^{(7)}$. However, there are some assumptions and limitations in this approach that should be considered. The method does not allow to study LCPUFA retroconversion. Likewise, fatty acid $\beta$-oxidation is estimated based on the fatty acid disappearance, while the use of fatty acids for the synthesis of non-fatty acid metabolites, e.g. hormones or PG, is not considered (though quantitatively extremely limited). However, it is important to underline that other methods employing labelled fatty acid techniques are accomplished with similar assumptions. The main difference between the WB method and other methods used for fatty acid metabolism studies (e.g. perfused isolated liver isotopical study) is the time frame ${ }^{(14,29)}$. In fact, the WB method needs a longer time frame compared with the other methods. Consequently, the average enzyme activity (enzyme velocity relative to total enzyme product over time) is estimated in a longer time frame. Therefore, estimated apparent enzyme activity may deviate from the instantaneous enzyme activity measured at a given time, but it likely provides useful information relative to whole metabolism in growing animals, resulting in a reliable estimation of the apparent in vivo metabolic activity.

In consideration of the fact that $n-3$ LCPUFA (namely $20: 5 n-3,22: 5 n-3$ and $22: 6 n-3)$ are reportedly beneficial to human health ${ }^{(1,3,5)}$, there is great interest towards finding possible ways to increase the $n$ - 3 LCPUFA content of farmed animals. In the present study, we recorded that in broiler chickens fed the Lin diet for $42 \mathrm{~d}$, hence receiving the highest amount of $18: 3 n-3$, a total of $5.8 \%$ of the net intake of $18: 3 n-3$ was eventually converted to $n-3$ LCPUFA. On a molar basis, the conversion of $18: 3 n-3$ to $20: 5 n-3$ and $22: 6 n-3$ was 2.7 and $3.3 \%$ on the Palm diet, 1.6 and $2.7 \%$ on the Soya diet and 2.0 and $1.2 \%$ on the Lin diet, respectively. In rainbow trout fed a Lin-based diet, it was reported that $8.8 \%$ of the net $18: 3 n-3$ dietary intake was bioconverted to $n-3$ LCPUFA $^{(14)}$. Thus, in comparison to the values reported in human subjects ${ }^{(4,5)}$, the $18: 3 n-3$ to $n$-3 LCPUFA bioconversion capacity of broiler chicken and rainbow trout appears to be remarkably greater, particularly the synthesis of $22: 6 n-3$. Two important conclusions can be obtained from this observation. Generalising, it can be speculated that the efficiency of the bioconversion of $18: 3 n-3$ to $n-3$ LCPUFA seems to be inversely related to evolution (rainbow trout $>$ broiler chicken $>$ human). Secondly, the inclusion of Lin (or other sources of $18: 3 n-3$ ) in animal feed results in beneficial net production of $n-3$ LCPUFA. Considering breast and thigh meat (without skin) as the only edible parts of the chicken carcass, and using their proportion of the body weight and their fatty acid composition ${ }^{(9)}$, it was calculated that $100 \mathrm{~g}$ of meat (breast and thigh) provided $62 \mathrm{mg} \mathrm{n}-3$ LCPUFA $(20: 5 n-3,22: 5 n-3$ and $22: 6 n-3)$ on the Lin diet. On the other hand, the actual total intake of $18: 3 n-3$ to produce the live biomass corresponding to $100 \mathrm{~g}$ of edible meat was $7581 \mathrm{mg}$. On the Fish diet, $100 \mathrm{~g}$ meat provided $198 \mathrm{mg} n$-3 LCPUFA, corresponding to an intake by the broiler chickens directly of $4284 \mathrm{mg} n-3$ LCPUFA. Although the supply of $n$-3 LCPUFA was 3-fold higher in meat from Fish fed chicken compared with Lin fed chicken, the latter meat did not require consumption of $n-3$ LCPUFA by the broiler chicken. Thus, from a human nutrition viewpoint, the present data suggest that it is more beneficial to consume chicken fed Lin than the practice of direct consumption of Lin or other sources of $18: 3 n-3$ alone by human subjects, in consideration that the conversion efficiency of $18: 3 n-3$ to $n-3$ LCPUFA in human subjects is lower than $0.80 \%{ }^{(30)}$. Additionally, from an environmental viewpoint, the use of fish oil in chicken nutrition seems to be a relatively wasteful practice of this precious and limited natural resource.

In conclusion, the metabolism (elongation, desaturation and $\beta$-oxidation) of PUFA in broiler chickens is mainly affected by the dietary fatty acid source rather than the animal factors. Dietary provision of $18: 3 n-3$ promotes the apparent elongation and desaturation activity on fatty acids in the $n-3$ pathway. Hence, $18: 3 n-3$ bioconversion relative to the net intake of this fatty acid seems to be limited by the dietary supply. Feeding fish oil suppresses apparent elongase and 
desaturase activity, but it was responsible for significantly higher total deposition of $n-3$ LCPUFA. Apparent elongase and desaturase activity appeared to be higher in young broiler chickens $(21 \mathrm{~d})$ compared with those at conventional slaughter age (42 d). Sex has only a marginal effect on the PUFA metabolism in broiler chicken.

\section{Acknowledgements}

The authors wish to acknowledge the Iranian Ministry of Science and Technology for providing the first author the opportunity to do this research. The technical staff of the Laboratory of Animal Nutrition and Animal Product Quality and of the Animal Science Unit of the Institute for Agricultural and Fisheries Research involved in this experiment are gratefully thanked for their skilled assistance. None of the authors has any financial or personal conflict of interest to disclose. The study was conducted by own funding. R. P. conducted the laboratory analysis and was involved in data analysis and writing of the manuscript. G. M. T. assisted in data analysis and manuscript writing. G. H. was involved in setting up and carrying out the animal experiment. K. R. and S. D. S. were involved in the experimental setup, data analysis and manuscript writing.

\section{References}

1. Ruxton CHS, Calder PC, Reed SC, et al. (2005) The impact of long-chain $n-3$ polyunsaturated fatty acids on human health. Nutr Res Rev 18, 113-129.

2. Leonard AE, Pereira SL, Sprecher H, et al. (2004) Elongation of long-chain fatty acids. Prog Lipid Res 43, 36-54.

3. Griffin BA (2008) How relevant is the ratio of dietary $n-6$ to $n-3$ polyunsaturated fatty acids to cardiovascular disease risk? Evidence from the OPTILIP study. Curr Opin Lipidol 19, 57-62.

4. Burdge GC \& Calder PC (2005) Conversion of $\alpha$-linolenic acid to longer-chain polyunsaturated fatty acids in human adults. Reprod Nutr Dev 45, 581-597.

5. Brenna JT, Salem N, Sinclair AJ, et al. (2009) $\alpha$-Linolenic acid supplementation and conversion to $n-3$ long-chain polyunsaturated fatty acids in humans. Prostaglandins Leukot Essent Fatty Acids 80, 85-91.

6. Brown JE (2005) A critical review of methods used to estimate linolenic acid $\Delta-6$ desaturase ex vivo and in vivo. Eur J Lipid Sci Technol 107, 119-134.

7. Turchini GM, Francis DS \& De Silva SS (2007) A whole body, in vivo, fatty acid balance method to quantify PUFA metabolism (desaturation, elongation and beta-oxidation). Lipids $\mathbf{4 2}$, 1065-1071.

8. Cunnane S (2001) Application of new methods and analytical approaches to research on polyunsaturated fatty acid homeostasis. Lipids 36, 975-979.

9. Poureslami R, Raes K, Huyghebaert G, et al. (2009) Effect of diet, age, and gender on the polyunsaturated fatty acid composition of broiler anatomical compartments. $\mathrm{Br}$ Poult Sci (In the Press).

10. Folch J, Lees M \& Sloane Stanley GH (1957) A simple method for the isolation and purification of total lipides from animal tissues. J Biol Chem 226, 497-509.

11. Raes K, De Smet S \& Demeyer D (2001) Effect of doublemuscling in Belgian blue young bulls on the intramuscular fatty acid composition with emphasis on conjugated linolenic acid and polyunsaturated fatty acids. J Anim Sci 73, 253-260.
12. Ackman GR (2002) The gas chromatograph in practical analyses of common and uncommon fatty acids for the $21 \mathrm{st}$ century. Anal Chim Acta 465, 175-192.

13. Turchini GM, Francis DS \& De Silva SS (2008) A whole body, in vivo, fatty acid balance method to quantify PUFA metabolism (desaturation, elongation and beta-oxidation). Erratum to Lipids (2007). Lipids 43, 977.

14. Turchini GM \& Francis DS (2009) Fatty acid metabolism (desaturation, elongation and $\beta$-oxidation) in rainbow trout fed fish oil- or linseed oil-based diets. Br J Nutr 102, 69-81.

15. Krogdahl A (1985) Digestion and absorption of lipids in poultry. J Nutr 115, 675-685.

16. Crespo N \& Esteve-Garcia E (2002) Nutrient and fatty acid deposition in broilers fed different dietary fatty acid profiles. Poult Sci 81, 1533-1542.

17. Smink W, Gerrits WJJ, Hovenier R, et al. (2008) Fatty acid digestion and deposition in broiler chickens fed diets containing either native or randomized palm oil. Poult Sci 87, 506-513.

18. Kloareg M, Bellego LL, Mourot J, et al. (2005) Deposition of dietary fatty acids and of de novo synthesis in growing pigs: effect of high ambient temperature and feeding restriction. Br J Nutr 93, 803-811.

19. Turchini GM, Francis DS \& De Silva SS (2006) Fatty acid metabolism in the freshwater fish Murray cod (Maccullochella peelii peelii) deduced by the whole-body fatty acid balance method. Comp Biochem Physiol B Biochem Mol Biol 144, $110-118$.

20. Vermunt SHF, Mensink RP, Simonis AMG, et al. (2000) Effects of dietary $\alpha$-linolenic acid on the conversion and oxidation of $\left[{ }^{13} \mathrm{C}\right]-\alpha$-linolenic acid. Lipids 35, 137-142.

21. Miller MR, Bridle AR, Nichols PD, et al. (2008) Increased elongase and desaturase gene expression with stearidonic acid enriched diet does not enhance long-chain (n-3) content of seawater Atlantic salmon (Salmon salar L.). J Nutr 138, 2179-2185.

22. Marra CA \& de Alaniz MJ (1989) Influence of testosterone administration on the biosynthesis of unsaturated fatty acids in male and female rats. Lipids 24, 1014-1019.

23. Burdge GC \& Wootton SA (2002) Conversion of $\alpha$-linolenic acid to eicosapentaenoic, docosapentaenoic and docosahexaenoic acids in young women. Br J Nutr 88, 411-420.

24. Childs CE, Romeu-Nadal M, Burdge GC, et al. (2008) Gender differences in the $n-3$ fatty acid content of tissues. Proc Nutr Soc 67, 19-27.

25. Jones AE, Murphy JL, Stolinski M, et al. (1998) The effect of age and gender on the metabolic disposal of $\left[1-{ }^{13} \mathrm{C}\right]$ palmitic acid. Eur J Clin Nutr 52, 22-28.

26. Leyton J, Drury PJ \& Crawford MA (1987) Differential oxidation of saturated and unsaturated fatty acids in vivo in rat. Br J Nutr 57, 383-393.

27. Burdge GC, Jones AE \& Wootton SA (2002) Eicosapentaenoic and docosahexaenoic acids are the principle products of $\alpha$-linolenic acid metabolism in young men. Br J Nutr 88, 355-363.

28. Kloareg M, Noblet J \& Milgen JV (2007) Deposition of dietary fatty acids, de novo synthesis and anatomical partitioning of fatty acids in finishing pigs. Br J Nutr 97, 35-44.

29. Francis DS, Peters DJ \& Turchini GM (2009) Apparent in vivo $\Delta-6$ desaturase activity, efficiency, and affinity are affected by total dietary $\mathrm{C}_{18}$ PUFA in the freshwater fish Murray cod. J Agric Food Chem 57, 4381-4390.

30. Wang C, Harris WS, Chung M, et al. (2006) n-3 Fatty acids from fish or fish-oil supplements, but not $\alpha$-linolenic acid, benefit cardiovascular disease outcomes in primary- and secondaryprevention studies: a systematic review. Am J Clin Nutr 84, $5-17$. 